\title{
THE INFRARED COUNTERPART OF THE MICROQUASAR GRS 1758-258
}

\author{
D. M. Rothstein, ${ }^{1}$ S. S. Eikenberry, ${ }^{1}$ S. Chatterjee, ${ }^{1}$ E. Egami, ${ }^{2}$ S. G. Djorgovski, ${ }^{3}$ and W. A. Heindl ${ }^{4}$ \\ Received 2002 June 14; accepted 2002 October 15; published 2002 October 23
}

\begin{abstract}
We present revised infrared $(2.2 \mu \mathrm{m})$ astrometry of the field containing the Galactic microquasar GRS 1758-258, using observations at the Keck I $10 \mathrm{~m}$ telescope. We find three candidates for the microquasar within a $3 \sigma$ error circle but none within $2 \sigma$. We show that if the 18.4 day X-ray period of GRS $1758-258$ is due to a binary orbit, then only one of the three candidates, an early K-type giant, is large enough to power the microquasar via Roche lobe overflow. We therefore identify this star as the infrared counterpart of GRS 1758-258, which we classify as a low-mass X-ray binary. Long-term infrared monitoring of this source should provide further information about the microquasar system, including a confirmation of the X-ray period and an estimate of the compact object's mass.
\end{abstract}

Subject headings: black hole physics — infrared: stars — stars: individual (GRS 1758-258) — X-rays: stars

\section{INTRODUCTION}

GRS $1758-258$ was one of the first objects in the Galaxy to be identified as a "microquasar"- an X-ray binary with jets whose behavior mimics quasars on a smaller and closer scale. Because timescales in microquasars are a factor of $\sim 10^{8}$ shorter than quasars, microquasars are excellent laboratories for investigating the jet formation process in compact objects.

The status of GRS $1758-258$ as a microquasar was cemented by the discovery of radio lobes indicating the presence of collimated jet outflows (Rodríguez, Mirabel, \& Martí 1992). It is one of the two brightest hard X-ray sources near the Galactic center at energies greater than $50 \mathrm{keV}$ (Sunyaev et al. 1991), and its hard X-ray spectra and variability are similar to that of Cyg X-1 (Kuznetsov et al. 1996), a black hole candidate that accretes via the stellar wind from its $\mathrm{O}$ supergiant companion.

However, the behavior of GRS 1758-258 during the transition between hard and soft spectral states is markedly different from that of Cyg X-1: there is an observed time delay of $\sim 1$ month between changes in its luminosity and spectral hardness. This suggests that GRS 1758-258 has two separate accretion flows, a thin disk and a halo, and that the observed time delay is equal to the viscous timescale of the thin disk. In this case, the calculated disk radius $\left(3 \times 10^{10} \mathrm{~cm}\right)$ is too large for a wind-fed system and instead indicates that GRS 1758-258 has a lowmass companion and is powered by Roche lobe overflow (Main et al. 1999; Smith et al. 2001; Smith, Heindl, \& Swank 2002a).

Because of the high optical obscuration $\left(A_{V} \sim 10\right)$ along the line of sight to GRS 1758-258, searches for companions have been mostly restricted to the infrared. Early attempts did not find any candidates, but massive supergiants were quickly ruled out (Chen, Gehrels, \& Leventhal 1994; Mereghetti et al. 1997). Martí et al. (1998) found three obscured optical stars in or near the radio error circle, but owing to the crowded nature of the field, none of the candidates could be definitively identified as the microquasar counterpart.

We (Eikenberry et al. 2001) obtained deep $K_{s}(2.15 \mu \mathrm{m}) \mathrm{im}$ -

\footnotetext{
${ }^{1}$ Department of Astronomy, Cornell University, 304 Space Sciences Building, Ithaca, NY 14853; droth@astro.cornell.edu.

${ }^{2}$ Steward Observatory, University of Arizona, 933 North Cherry Avenue, Tucson, AZ 85721.

${ }^{3}$ Palomar Observatory, California Institute of Technology, MS 105-24, Pasadena, CA 91125.

${ }^{4}$ Center for Astrophysics and Space Sciences, University of California, San Diego, Code 0424, 9500 Gilman Drive, La Jolla, CA 92093.
}

ages of the field and found an $\sim 1 " .5$ offset between our derived position for GRS $1758-258$ and the one obtained by Martí et al. (1998). Here we report an error in our original astrometry and obtain a revised infrared position of GRS 1758-258 consistent with that of Martí et al. (1998). The apparent $18.45 \pm$ 0.10 day X-ray orbital period of the microquasar (Smith, Heindl, \& Swank 2002b), combined with the requirement that the system undergo Roche lobe overflow, imposes a constraint on the size of the companion, which allows us to identify one of the stars first seen by Martí et al. (1998) — an early K-type giant referred to as "star A" in Eikenberry et al. (2001)—as the infrared counterpart of GRS $1758-258$.

\section{OBSERVATIONS AND DATA REDUCTION}

We present a brief description of the observations, which are discussed in more detail by Eikenberry et al. (2001).

We observed the field containing GRS $1758-258$ for a total of 94 minutes on 1998 June 1 UTC and 99 minutes on June 2 with the Keck I telescope, using a $K_{s}$ filter (centered at $2.15 \mu \mathrm{m}$ ) on the Keck Observatory's Near-Infrared Camera (NIRC; Matthews \& Soifer 1994). NIRC has a $256 \times 256$ pixel InSb array with a 0.15 pixel $^{-1}$ plate scale, giving a $38^{\prime \prime}$ field of view. The seeing conditions were exceptionally good, ranging from $\sim 0$ ".35 to $\sim 0$ ".55, with extended periods of seeing less than 0.5. All images obtained were shifted and combined to form a master image of the GRS 1758-258 field.

In order to accurately calibrate the astrometric reference frame, we obtained wide-field $\left(8^{\prime} \times 8^{\prime}\right)$ CCD images of this field in the $I$ band on 1998 June 29 using the Palomar $1.5 \mathrm{~m}$ (60 inch) telescope. The total exposure time was $300 \mathrm{~s}$, and the typical seeing was $\sim 1 " .2$.

We performed astrometry on these images using nine stars listed in the USNO-A2.0 astrometric catalog (Monet et al. 1998) that were visible in the I-band CCD image within $~ 1.5$ of the radio position of GRS 1758-258 and that did not appear to be double stars, extended sources, or in extremely crowded regions. There were no suitable USNO stars visible in the $K_{s}$ band NIRC image itself, so we obtained a best-fit astrometric solution for the CCD image using the above nine USNO stars, determined the coordinates of five stars near the position of GRS 1758-258 that were visible in both the CCD and NIRC images, and thereby derived a secondary astrometric solution for the NIRC image.

Figure 1 shows a portion of the NIRC image with the resulting 


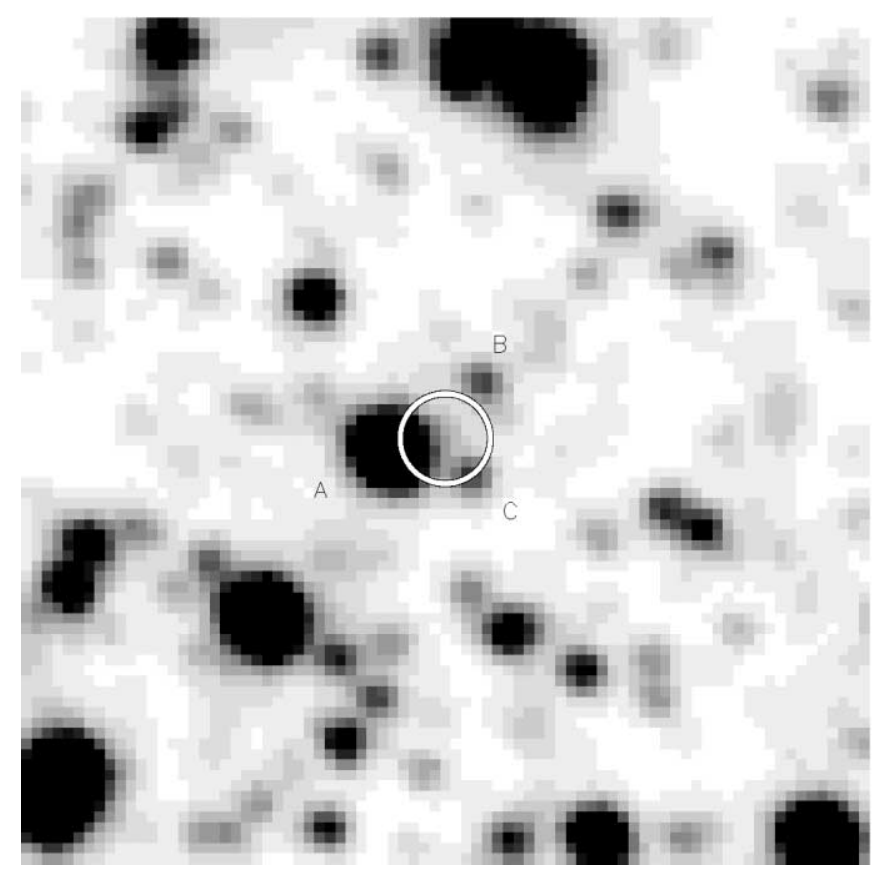

FIG. 1.-Close-up $\left(12^{\prime \prime} \times 12^{\prime \prime}\right)$ Keck $K_{s}$-band image of the field of GRS $1758-258$. North is up, and east is to the left. Our $2 \sigma$ error circle (1".3 diameter) for the microquasar position is shown; it is virtually identical to the error circle obtained in Fig. 2 of Martí et al. (1998). The stars labeled A, B, and C are discussed further in the text.

position of GRS 1758-258. We used the Very Large Array (VLA) position $\alpha(\mathrm{J} 2000.0)=18^{\mathrm{h}} 01^{\mathrm{m}} 12^{\mathrm{s}} 395, \delta(\mathrm{J} 2000.0)=$ $-25^{\circ} 44^{\prime} 35^{\prime \prime} .90$ (Mirabel \& Rodríguez 1993), which we have confirmed through a reanalysis of the VLA data. This is also consistent with the VLA position determined by Martí et al. (2002) using more recent observations and with the X-ray position determined by Chandra (Heindl \& Smith 2002).

Our $2 \sigma$ error circle of radius 0 ."64 was determined by combining the $00^{\prime \prime} 1$ uncertainty in the radio position (Mirabel \& Rodríguez 1993), the less than 0"1 uncertainty in the radiooptical frame tie (da Silva Neto et al. 2000), and our calculated rms residuals for the CCD astrometric solution (0"28) and the CCD to infrared solution ( $\left.00^{\prime \prime} 05\right)$. We note that the accuracy of the radio-optical frame tie in this region of the sky may be worse than the nominal value (because of the paucity of quasars in the Galactic plane), so our $2 \sigma$ error circle may be an underestimate. The GRS $1758-258$ position obtained here is consistent with that of Martí et al. (1998) — the discrepancy reported in Eikenberry et al. (2001) was due to a transcription error in shifting CCD images while performing the astrometry for that paper.

We find no sources within the $2 \sigma$ error circle, but three sources appear within $3 \sigma$-those labeled A, B, and C in Figure 1. Star $A$ is at a distance of 0 ".8 from the GRS $1758-258$ position, star $B$ is at 0.9 , and star $C$ is at 0.7 . At the center of the GRS 1758-258 error circle, the pixel-to-pixel variation is heavily dominated by background gradients from the nearby stars, but we estimate an upper limit of $m_{K_{s}} \lesssim 18$, at the $95 \%$ confidence level, on any star not detected.

Eikenberry et al. (2001) performed photometry on stars A, $\mathrm{B}$, and $\mathrm{C}$ in the $K_{s}$ band and estimated their absolute magnitudes assuming an extinction of $A_{K_{s}}=0.9 \mathrm{mag}$ (derived from the neutral hydrogen column density along the line of sight to GRS 1758-258; Mereghetti et al. 1997) and a distance of $8.5 \mathrm{kpc}$. They found that star A is consistent with an early K-type gi-

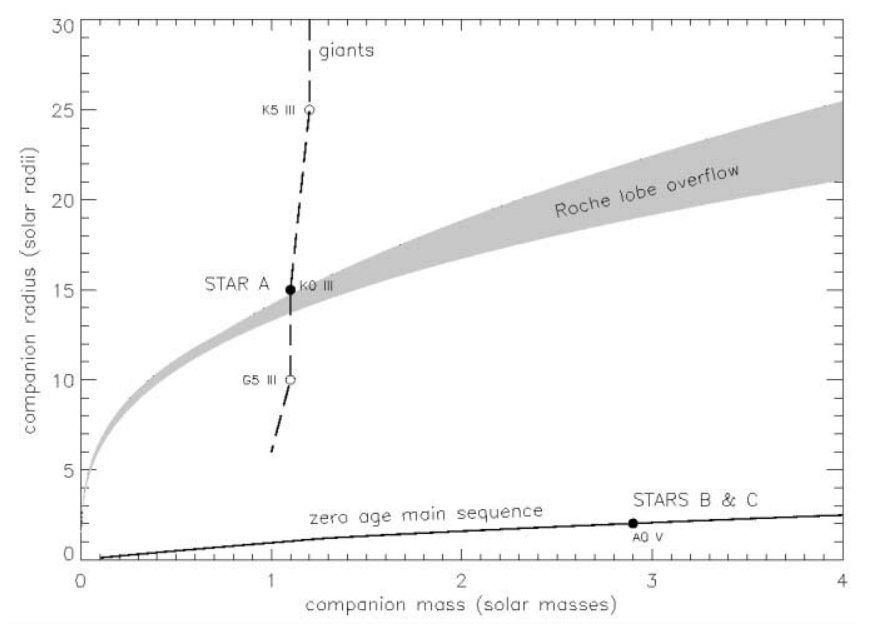

FIG. 2.-Possible values of the companion radius for the GRS 1758-258 system, as a function of companion mass. The shaded region represents the minimum radius required to undergo Roche lobe overflow, for compact object masses between 1 and $30 M_{\odot}$ and orbital periods within $18.45 \pm 0.30$ days. The solid line shows the mass-radius relation for zero-age main-sequence stars, and the dashed line shows the mass-radius relation for giants, with the approximate locations of stars A, B, and C overplotted (see text and Fig. 1).

ant—as proposed by Martí et al. (1998) based on multiband photometry and near-infrared spectroscopy-and stars B and $\mathrm{C}$ are consistent with early A-type main-sequence stars.

\section{DISCUSSION}

In the following discussion, we assume that the X-ray emission from GRS $1758-258$ is powered by accretion via Roche lobe overflow (Main et al. 1999; Smith et al. 2001, 2002a) and that its $18.45 \pm 0.10$ day periodicity (Smith et al. 2002b) is due to a binary orbit.

For Roche lobe overflow to occur, the radius of the companion star must satisfy

$$
R_{s} \geq \frac{0.49 q^{2 / 3}}{0.6 q^{2 / 3}+\ln \left(1+q^{1 / 3}\right)} a,
$$

where $q$ is the mass ratio of the companion to the compact object and $a$ is the binary separation (Eggleton 1983). Assuming a circular orbit and the period given above, we can eliminate $a$ and find the minimum value of $R_{s}$ in terms of the companion and compact object masses.

The shaded region of Figure 2 shows the minimum values of $R_{s}$ required for Roche lobe overflow, as a function of companion mass, for compact objects between 1 and $30 M_{\odot}$ and periods within $3 \sigma$ of 18.45 days. Also shown is the massradius relation for the zero-age main sequence and for giants (Drilling \& Landolt 2000), with the approximate locations of our stars A, B, and C marked for clarity.

It is clear that star A, the early K-type giant, is the only one consistent with Roche lobe overflow. Even in the case of an elliptical orbit, an eccentricity $\gtrsim 0.9$ would be required for stars $\mathrm{B}$ or $\mathrm{C}$ to undergo brief periods of Roche lobe overflow at their minimum orbital separation, and there is no evidence in the X-ray light curve of GRS 1758-258 for such intermittent behavior. Any other star large enough to undergo Roche lobe overflow in this system would have been detected within our astrometric error circle if it were located within the Galaxy-for example, a K0 giant would need to be farther than $60 \mathrm{kpc}$ to fall below our detection limit of $m_{K_{s}}=18$ (assuming $A_{K_{s}}=$ 
0.9). We therefore identify star A as the infrared counterpart of GRS $1758-258$.

\section{CONCLUSIONS}

We have presented revised infrared $(2.2 \mu \mathrm{m})$ astrometry of the field containing the Galactic microquasar GRS 1758-258. We summarize our results as follows:

1. We find three candidates within a $3 \sigma$ error circle of the microquasar position, which is consistent with the results obtained by Martí et al. (1998).

2. Assuming an $18.45 \pm 0.10$ orbital period of the GRS 1758-258 system (Smith et al. 2002b), we calculate the radius of the companion star required to undergo Roche lobe overflow for a range of companion and compact object masses.

3. We find that only one of our candidate stars (an early K-type giant labeled star A in Fig. 1) is consistent with Roche lobe overflow, and we therefore identify this star as the infrared counterpart of GRS 1758-258.

Finally, we note that there are strong ${ }^{12} \mathrm{C}^{16} \mathrm{O}$ absorption bands (equivalent widths $\sim 10 \AA$ ) in the infrared spectrum of the can- didate (Martí et al. 1998). Long-term monitoring of these lines should provide a determination of the GRS 1758-258 mass function. This would allow us to constrain the mass of the compact object and determine whether it is a neutron star or, as suspected from its similarity to Cyg X-1, a black hole.

This Letter is based in part on observations obtained at the W. M. Keck Observatory, which is operated by the California Association for Research in Astronomy, a scientific partnership among the California Institute of Technology, the University of California, and the National Aeronautics and Space Administration. The VLA is a facility of the National Radio Astronomy Observatory, a facility of the National Science Foundation operated under cooperative agreement by Associated Universities, Inc. The authors thank L. Armus and R. Gal for help in obtaining the CCD images at Palomar Observatory. We also thank the staff at Palomar and Keck Observatories for their expert help during our observing runs. D. M. R. is supported in part by a National Science Foundation Graduate Research Fellowship. S. S. E. and D. M. R. are supported in part by an NSF CAREER award (NSF-9983830).

\section{REFERENCES}

Chen, W., Gehrels, N., \& Leventhal, M. 1994, ApJ, 426, 586

da Silva Neto, D. N., Andrei, A. H., Martins, R. V., \& Assafin, M. 2000, AJ, 119,1470

Drilling, J. S., \& Landolt, A. U. 2000, in Allen's Astrophysical Quantities, ed. A. N. Cox (4th ed.; New York: AIP), 381

Eggleton, P. P. 1983, ApJ, 268, 368

Eikenberry, S. S., Fischer, W. J., Egami, E., \& Djorgovski, S. G. 2001, ApJ, 556,1

Heindl, W. A., \& Smith, D. M. 2002, ApJ, 578, L125

Kuznetsov, S., et al. 1996, in Proc. Roentgenstrahlung from the Universe, ed. H. U. Zimmermann, J. Trümper, \& H. Yorke (MPE Rep. 263; Garching: MPE), 157

Main, D. S., Smith, D. M., Heindl, W. A., Swank, J., Leventhal, M., Mirabel, I. F., \& Rodríguez, L. F. 1999, ApJ, 525, 901

Martí, J., Mereghetti, S., Chaty, S., Mirabel, I. F., Goldoni, P., \& Rodríguez, L. F. 1998, A\&A, 338, L95
Martí, J., Mirabel, I. F., Rodríguez, L. F., \& Smith, I. A. 2002, A\&A, 386, 571

Matthews, K., \& Soifer, B. T. 1994, Exp. Astron., 3, 77

Mereghetti, S., Cremonesi, D. I., Haardt, F., Murakami, T., Belloni, T., \& Goldwurm, A. 1997, ApJ, 476, 829

Mirabel, I. F., \& Rodríguez, L. F. 1993, in AIP Conf. Proc. 304, The Second Compton Symposium, ed. C. E. Fichtel, N. Gehrels, \& J. P. Norris (New York: AIP), 413

Monet, D. G., et al. 1998, USNO-A2.0, A Catalog of Astrometric Standards (Washington, DC: USNO)

Rodríguez, L. F., Mirabel, I. F., \& Martí, J. 1992, ApJ, 401, L15

Smith, D. M., Heindl, W. A., Markwardt, C. B., \& Swank, J. H. 2001, ApJ, 554, L41

Smith, D. M., Heindl, W. A., \& Swank, J. H. 2002a, ApJ, 569, 362

. 2002b, ApJ, 578, L129

Sunyaev, R., et al. 1991, ApJ, 383, L49 